\title{
Índices de vulnerabilidad de redes carreteras. Enfoques recientes y propuesta de aplicación en México
}

\author{
Measures of Road Network Vulnerability. \\ Recent Approaches and a Proposed Application in Mexico
}

\author{
Gradilla-Hernández L.A. \\ Facultad de Ingeniería \\ Universidad Autónoma de Querétaro \\ E-mail:luzgradilla@gmail.com \\ De la Llata-Gómez R. \\ Centro Queretano de Recursos Naturales \\ Consejo de Ciencia y Tecnología del Estado de Querétaro \\ E-mail: rllata@concyteq.org.mx
}

\author{
González-Gómez O. \\ División de Posgrado Facultad de Ingeniería \\ Universidad Autónoma de Querétaro \\ E-mail:ovidio@uaq.mx
}

Información del artículo: recibido: enero de 2009, aceptado: febrero de 2010

\section{Resumen}

En este artículo se propone la integración de una metodología de análisis multicriterio para identificar tramos críticos de redes carreteras, que pueda ser aplicada en México. Para ello, se hizo una revisión de trabajos recientes sobre vulnerabilidad de redes carreteras, cuyos avances principales son analizados aquí. Se encontró un concepto de vulnerabilidad, aceptado por varios autores, relacionado con las consecuencias de la falla de un tramo, independientemente de la probabilidad de que dicha falla ocurra. En algunos de los trabajos citados se propone que se tome en cuenta la capacidad de los tramos carreteros en el análisis de vulnerabilidad, por lo cual ésta se incluye en la metodología propuesta. Finalmente, se realiza un ejercicio a partir de una red hipotética y se esboza la forma que tomaría el modelo multicriterio y la determinación de las importancias relativas de los criterios.

\footnotetext{
Abstract

In this paper, the integration of a multi-criteria analysis methodology that enables identification of critical road network links that can be applied to Mexico is proposed. In order to achieve this, recent works about road network vulnerability are reviewed in this article and their main findings are analyzed. It was found a concept of vulnerability accepted by some authors, where vulnerability is related to the consequences of link failure, irrespective of the probability of such failure. Some cited works, propose to take into account the road link capacity in vulnerability analysis, for that reason it is included in the proposed methodology. Finally, an exercise using a hypothetical network is made and a multi-criteria model is described.
}

\section{Descriptores}

- sistema de transporte

- red carretera

- fallas de tramos carreteros

- tramo crítico

- análisis de vulnerabilidad

- análisis multicriterio

\section{Keywords}

- transport system

- road network

- link interruptions

- vulnerability analysis

- critical link

- multi-criteria analysis 


\section{Introducción}

Desde los años noventa del siglo pasado, se ha mostrado interés por realizar investigación que permita conocer y medir las redes de transporte automotor desde la perspectiva de su vulnerabilidad y confiabilidad. Dicho interés crece dado el incremento constante en la cuantía del valor movido de las mercancías en tránsito y por las consecuencias críticas de pérdida de tiempo (e incluso vidas) en el traslado de las personas. Recientemente, la conveniencia de contar con estimaciones realistas sobre las afectaciones en la red carretera ante fallas posibles de diverso tipo en algunos de sus tramos ha adquirido importancia crucial, sobre todo después de acontecimientos catastróficos como el terremoto de 1995 en Kobe, Japón y los ataques terroristas del 11 de septiembre de 2001 en Estados Unidos (Di Gangi et al., 2005; Husdal, 2006; Jenelius et al., 2006).

La mayoría de los autores que recientemente han tratado este tema (Jenelius et al., 2006; Berdica, 2002a; Lleras et al., 2001; Tampère et al., 2007) coinciden en que el término vulnerabilidad debería enfocarse a los efectos de las amenazas sobre la infraestructura de transporte; sin embargo, el concepto de vulnerabilidad de redes carreteras todavía no cuenta con una definición acabada y mucho menos, con una expresión matemática aceptada (Husdal, 2006). El concepto de vulnerabilidad se ha relacionado con las consecuencias observables en la red, debidas a la falla de un tramo carretero, independientemente de su probabilidad de ocurrencia; es decir, se enfoca en el nivel de funcionamiento del sistema de transporte y en especial, en la medición de los posibles cambios en la conectividad o costo generalizado, provocados por eventos tales como desastres naturales, clima adverso, accidentes vehiculares, etcétera.

Las interrupciones en los sistemas de transporte pueden implicar graves pérdidas económicas, ya que la obstrucción en uno o más tramos carreteros provoca que se exceda la capacidad de otros tramos y como consecuencia, el tráfico ha de ser redirigido con un alto costo. Por lo general, en las redes urbanas existen más rutas alternas que en las carreteras regionales o nacionales si queda obstruido un tramo dentro de una ruta óptima, de forma tal que una falla similar puede provocar efectos más drásticos en las redes regionales que en las urbanas. Una primera aproximación al concepto de vulnerabilidad tiene que ver, entonces, con la densidad de la red. En la medida que la red es más densa, el sistema resulta menos vulnerable y al revés, una red con escasas conexiones redundantes provoca un grado de vulnerabilidad mayor en su sistema de transporte.
En Latinoamérica, este grado de afectación o vulnerabilidad de las redes carreteras se incrementa, dado que existe una mayor dependencia operativa (y por tanto económica) en algunas rutas carreteras (sin alternativas redundantes) que, en su mayoría y por la historia de dependencia económica, pertenecen a los senderos a través de los cuales se realizan los intercambios mercantiles hacia el exterior.

$\mathrm{Al}$ incrementarse estos intercambios con el proceso de globalización, más severo puede ser el problema económico derivado del bloqueo de algún tramo, por lo que una metodología para identificar los tramos críticos o estratégicos de dichas rutas sería de gran utilidad. Sin embargo, la reducción del concepto de vulnerabilidad a solamente las características morfológicas de la red es poco realista, pues no toma en cuenta la interacción de la infraestructura carretera con los flujos vehiculares que de manera dinámica se readaptan a condiciones cambiantes de la operación y lo que aquí interesa, a las modificaciones abruptas en la morfología de la red, esto es, a fallas en algún(os) tramo(s).

La vulnerabilidad es un concepto que hace referencia a los efectos provocados por la falla de algún(os) tramo(s) del sistema de transporte carretero en su conjunto. Sin embargo, existen tramos en particular con capacidad de crear un efecto grave en el sistema, a este tipo de tramos se les conoce en la literatura especializada como "tramos críticos".

El principal objetivo de medir la vulnerabilidad de redes carreteras estriba en proporcionar a los planificadores y tomadores de decisiones una de las herramientas necesarias para priorizar el mantenimiento y rehabilitación de los tramos carreteros críticos o la construcción de tramos redundantes que proporcionen rutas alternas, como resultado, optimizar recursos en países donde el transporte de carga se lleva a cabo mayoritariamente a través de la red de carreteras; obteniendo en consecuencia beneficios sociales y económicos.

En el siguiente apartado se revisan críticamente algunos enfoques para el análisis de vulnerabilidad de redes carreteras que comparten las bases de conceptualización de vulnerabilidad pero que difieren en las expresiones matemáticas o modelos utilizados para su cuantificación.

Es decir, la mayoría de los investigadores han propuesto índices y modelos para medir diversos tipos de consecuencias que provoca la falla de un tramo carretero, que son los casos aquí abordados, independientemente de la probabilidad de dicha falla; sin embargo, esos índices y/o modelos difieren entre sí. 


\section{Enfoques para el análisis de vulnerabilidad}

Los enfoques presentados en este apartado fueron agrupados en tres, de acuerdo a la similitud de las consecuencias que cuantifican. En el primer grupo, los autores revisados miden la conectividad; en el segundo, los cambios en accesibilidad y en el tercero, se agrupan los que ponen el énfasis en la medición de las consecuencias de la interrupción de un tramo carretero en función del tiempo y/o costo de viaje.

\section{Cambios en la conectividad}

Viswanath et al. (2003) sugirieron un concepto de vulnerabilidad de la red en su conjunto, en atención a la conexión redundante entre centroides y se mide por la suma del número de rutas independientes entre cada par origen-destino de la red de carreteras bajo estudio (una ruta independiente difiere en todos sus tramos carreteros de otras rutas y por lo tanto, provee redundancia). Mientras mayor sea esta sumatoria, menor será la vulnerabilidad de la red en su conjunto. Por los objetivos del trabajo de estos autores, la medida se propone para calcular el grado de vulnerabilidad de toda la red bajo estudio y no especifica estimación alguna de qué tan crítico sea cada tramo, por lo que la propuesta es insuficiente para planear y priorizar el mantenimiento, así como la rehabilitación después de un desastre de los tramos carreteros. Las redes carreteras en Latinoamérica podrían contar con pocas rutas independientes, debido a las escasas rutas carreteras que se utilizan para el trasporte doméstico e internacional de carga. Así, esta medida pudiera servir en las redes carreteras latinoamericanas para cuantificar con mayor precisión la calificación subjetiva de vulnerabilidad que se asume tienen, pero no para encaminar los esfuerzos de prevención por tramos.

Posteriormente, Di Gangi et al. (2005) presentaron una medida similar en perspectiva, pero más completa en alcance, pues atiende la debilidad de la red debida a cada uno de los tramos. Con esta medida, buscan determinar qué tan importante es cada tramo para el total de los pares origen-destino, dicha medida (ecuación 1) se obtiene con el cálculo del número de rutas origen-destino que comparten un mismo tramo, basado en modelos probabilísticos de elección de rutas. Entre más rutas O-D pasen por un mismo tramo, más rutas se verían afectadas por la obstrucción del tramo y se consideraría más crítico. En sí, no arroja un indicador de vulnerabilidad, sino una cuantificación relativa de la importancia de los tramos. La medida propuesta se enfoca a la medición de la conectividad, tomando en cuenta, así, sólo las características topológicas de la red, sin considera- ción a la circulación vehicular y, por tanto, sin referencia al posible efecto de sobrecongestión, debido a la obstrucción de un tramo.

$L W I_{i j}=\frac{\sum_{o d} N_{i j, o d} \cdot p_{i j, o d}}{\sum_{o d} N_{o d}^{k}}$

donde:

$L W I_{i j}=$ índice de debilidad del arco (Link Weakness Index),

$N_{i j, o d}=$ número de rutas que cruzan el arco $(\mathrm{i}, \mathrm{j})$ que conectan el par O-D,

$p_{i j, o d}=$ probabilidad de elección del arco $(\mathrm{i}, \mathrm{j})$, $N^{k}{ }_{o d}=$ número total de rutas que conectan el par O-D.

De forma similar, Murray et al. (2004) presentaron un modelo de programación matemática multiobjetivo para identificar los tramos carreteros críticos. En este modelo se propone un índice que mide la importancia que tiene un tramo específico para la conectividad entre un grupo de pares origen-destino y se toma en cuenta la disposición de rutas alternativas, el exceso de capacidad, así como el tiempo de viaje. Sin embargo, esta propuesta no ofrece una forma específica para volver operable el índice.

Este tipo de aproximaciones para medir directa o indirectamente la vulnerabilidad, a través de la calidad crítica de sus tramos, ofrece un muy importante avance en torno al manejo de la conectividad derivada de la topología de la red. Sin embargo, no sólo el primer modelo aquí descrito presenta limitaciones, sino que en su conjunto, estos modelos no incorporan ni permiten incorporar, tal como están planteados, la interacción de los flujos reales con la red.

Modificaciones en el acceso desde los nodos generadores

Una parte importante de los estudios de vulnerabilidad han sido desarrollados tomando como base que ésta debe estar relacionada con las consecuencias que tenga la obstrucción de uno o más tramos carreteros en el acceso de algunos o todos los nodos de la red. Si bien, la unidad de análisis es cada uno de los tramos, la atención está puesta en los efectos de la obstrucción en los nodos principales.

Así, se han desarrollado metodologías para medir la variación que provoca la obstrucción de uno o más tramos en los índices de accesibilidad (estándares o propuestos) de ciertas ciudades de interés en redes nacionales. 
Como primer caso, Lleras-Echeverri et al. (2001) propusieron medir los cambios en la accesibilidad nodal (ecuación 2), que ocasionaría cada escenario evaluado, es decir, la falla de uno o más de los tramos que se encuentran en los caminos más cortos entre los centroides analizados, para posteriormente calcular un parámetro de clasificación, $C P_{k}$, de tramos (ecuación 3) que permitiera identificar los que provocan mayores aumentos en el costo generalizado de viaje.

Es decir, estos autores centran su atención en la generación de escenarios complejos de falla, sin precisar la medida específica de accesibilidad que se utilizaría en la aplicación del modelo. Entonces, a pesar de que se menciona el "costo generalizado de viaje," éste es entendido meramente como la suma del tiempo y el costo directo de viaje (promedios) entre cada par origen destino del viaje, en condiciones de tráfico libre. Esto es, sin considerar la interacción de flujos con la red.

Para la construcción y evaluación de cada escenario $\mathrm{F}$, los autores desarrollaron un enfoque de fallas progresivas denominado escenario crítico (Critical Scenario) para el cual se evalúan los efectos de la obstrucción de " $s$ " tramos a la vez. Una de las reglas para detener la generación y evaluación de escenarios consiste en que el $C P_{k}$ de todos los tramos alcance un valor de variación $\varepsilon$, definido por el analista, entre un tipo de escenario y otro; cuando todos los valores $\mathrm{N}_{\mathrm{F}}$ alcanzan el valor de uno, también se detiene el análisis; de tal forma que se disminuyó el número total de escenarios evaluados. La metodología propuesta fue aplicada a un caso de estudio en una parte de la red carretera de Colombia. Sin embargo, el enfoque no toma en cuenta la demanda de viajes dentro de la red y en consecuencia, no se cubre la presencia de congestionamientos.

$\Delta A_{i F}=\frac{A_{i F}}{A_{i 0}}-1$

donde:

$A_{i F}=\quad$ accesibilidad del centroide i en el escenario de falla $\mathrm{F}$,

$A_{i 0}=\quad$ accesibilidad del centroide $\mathrm{i}$ en el escenario base 0 , en condiciones normales.

$C P_{k}=\sum_{s=1}^{m} f(s) \max \left(\frac{N_{F}}{\max \left(N_{F}\right)_{s}}\right)_{s}$

donde:

$\mathrm{CP}_{\mathrm{k}}=$ parámetro de clasificación del arco $\mathrm{k}$,
$\mathrm{N}_{F}=$ accesibilidad de la red,

$\mathrm{s}=$ número de tramos obstruidos al mismo tiempo.

En un trabajo posterior, Berdica et al. (2004) desarrollaron un indicador (ecuación 4) para determinar cómo se ve afectada la accesibilidad en una región determinada de Suecia cuando una parte de la red de carreteras, por alguna razón, queda completa o parcialmente deshabilitada para su uso. Si el valor $\Delta \Omega_{i}$ es negativo, indica un decremento en la accesibilidad.

$\Delta \Omega_{i}=\theta\left(\begin{array}{cc}-0 & -1 \\ t_{i} & -t_{i}\end{array}\right)+\left(\begin{array}{cc}-0 & -1 \\ c_{i} & -c_{i}\end{array}\right)+\bar{T}_{i}^{1}$

donde:

$\Delta \Omega_{i}=\quad$ cambio en accesibilidad para el área $i$, entre el escenario de falla 1 y el escenario en condiciones normales 0 ,

$t_{i}=$ tiempo promedio de viaje,

$c_{i}=$ costo promedio de viaje,

$T_{i}=$ viaje no realizado (supressed travel) comparado con un área de referencia o con la condición 0 .

Posteriormente, Taylor et al. (2006) presentaron otro enfoque para medir la vulnerabilidad de redes carreteras, en donde un tramo de red carretera es crítico si la obstrucción del tramo provoca una disminución significativa en la accesibilidad de determinadas ciudades, esta última medida con el índice ARIA (ecuación 5) que pondera la accesibilidad de acuerdo a la población y a las instalaciones de las ciudades de servicio; pero, al igual que en las metodologías antes descritas, no se toma en cuenta el congestionamiento y ni siquiera el funcionamiento de la red ante la demanda.

$A R I A_{i L}=\sum_{L} \min \left\{3, \frac{x_{i L}}{x_{L}}\right\}$

donde:

$A R I A_{i L}=$ índice de accesibilidad de Australia (Accessibility/Remoteness Index of Australia),

$\overline{x_{L}}=$ distancia media en carretera desde todas las localidades al centro de servicio más cercano de categoría $L$ (según sea el tamaño de población).

Ciertamente, estos modelos no miden de manera directa la vulnerabilidad de la red, aunque sí ofrecen estimaciones diversas de la afectación de una obstrucción en 
la accesibilidad, ya sea de la red completa o de la sumatoria de las accesibilidades de sus nodos generadores de viajes.

\section{Cambios en el costo de viaje}

Por otro lado, Jenelius et al. (2006) desarrollaron una medida (ecuación 6) para determinar la importancia de un arco k para el funcionamiento de la red, que toma en cuenta el incremento del costo generalizado de viaje, ponderado con la demanda insatisfecha cuando un arco individual está bloqueado. La medida se aplicó a un caso de estudio en el norte de Suecia. Sin embargo, se supone una demanda constante de viajes que puede provocar una sobreestimación de los problemas de congestionamiento después de un bloqueo en los arcos carreteros, porque no se considera que los usuarios podrían cambiar su decisión de viaje.

$$
\operatorname{Importancia}_{\text {red }}(k)=\frac{\sum_{i} \sum_{j \neq i} x_{i j}\left(c_{i j}^{(k)}-c_{i j}^{(0)}\right)}{\sum_{i} \sum_{j \neq i} x_{i j}}, k \in E^{n c}
$$

donde:

$\mathrm{c}_{\mathrm{ij}}^{(\mathrm{k})}=\quad$ costo de viaje, del nodo $i$ al $j$ cuando el arco $k$ ha fallado,

$\mathrm{c}_{\mathrm{ij}}{ }^{(0)}=$ costo de la red inicial, sin daño alguno,

$x_{i j}=$ demanda de viajes utilizada como peso para denotar la importancia del par origen-destino con relación a todos los demás pares de la red,

$\mathrm{E}^{\mathrm{nc}}=\quad$ conjunto de arcos que no dividen la red en partes desconectadas.

Berdica (2002b) reportó un caso de estudio en el cual se utiliza el modelo de asignación del software EMME/2 para estudiar la vulnerabilidad de la red carretera de Estocolmo. El estudio considera el comportamiento de elección de rutas solamente bajo condiciones de equilibrio y se miden los efectos en el tiempo de viaje, así como en las distancias recorridas en un nivel regional cuando se simulan escenarios en donde se obstruyen arcos de la red. Finalmente, se hace una estimación del costo total de la obstrucción de un arco a través del valor oficial de Suecia para el tiempo de viaje (official Swedish travel time value).

En forma similar, Scott et al. (2006) propusieron identificar los arcos críticos de una red carretera empleando el modelo de asignación del software Trans$\mathrm{CAD}^{\circledR}$ bajo el equilibrio del usuario y un índice (ecuación 7) que mide los cambios en el tiempo de viaje. La ventaja del enfoque radica en que el modelo empleado sí toma en cuenta la capacidad de los arcos y el he- cho de que los usuarios de la red que no utilizan el arco bloqueado pueden verse afectados por el congestionamiento provocado por los usuarios del arco bloqueado, que fueron redirigidos a otras rutas alternas, si éstas existen.

$$
N R I_{a}=c_{a}-c
$$

donde:

$N R I_{a}=\quad$ índice de robustez de la red (Network Robustness (ndex),

$c_{\mathrm{a}}=$ tiempo de viaje de todo el sistema cuando está inhabilitado el arco $a$,

$c=$ tiempo de viaje de todo el sistema cuando está presente la totalidad de los arcos (condiciones normales).

En contraste, Taylor et al. (2003 y 2004) propusieron un método para medir la vulnerabilidad a través de la aplicación de modelos probabilísticos de elección de rutas. Los autores presentaron una medida de vulnerabilidad (ecuación 8) que toma en cuenta los cambios del costo generalizado de viaje entre dos localidades si un arco carretero falla; sin embargo, no se toma en cuenta la capacidad de los arcos carreteros y por lo tanto, implícitamente se supone que no existe congestionamiento.

$V_{r s}=\sum_{i} \sum_{j} d_{i j} \cdot v_{i j r s}$

donde:

$V_{r s}=\quad$ pérdida para una región provocada por la falla del arco "rs",

$d_{i j}=$ demanda de viajes entre dos localidades $(i, j)$,

$v_{i j r s}=\quad$ cambio del costo generalizado de viajar entre dos puntos o localidades $(i, j)$ si un determinado $\operatorname{arco}\left(e_{r s}\right)$ falla.

En estos modelos, sí se ofrece un índice de vulnerabilidad de la red (robustez en el segundo caso), que permite comparar diferentes redes o distintas situaciones en una misma red. Sin embargo, se ha argumentado que se pueden obtener mejores estimaciones de los niveles de congestionamiento con la aplicación de modelos de asignación dinámica, al respecto, podemos mencionar el reciente trabajo de Tampère et al. (2007) en donde se aplican dichos modelos para determinar las secciones vulnerables de una red de carreteras, tomando en cuen- 
ta factores como el impacto en el tiempo de viaje que produce la obstrucción de una sección, en un periodo de tiempo dado.

La revisión de estos tipos de modelos lleva a considerar que mientras el último tipo de modelos acerca más a una medida satisfactoria, pues sí se toma en cuenta la operación del sistema de transporte, parecería aconsejable recoger también las ventajas de los otros tipos de modelos que se centran más en las características topológicas de la red y en las modificaciones de la capacidad de acceso de los nodos generadores de viajes, características que no se recogen en los últimos.

La virtud de los modelos en los que se considera sólo la pérdida de conectividad de la red estriba en que reflejan efectos de ruptura o reconfiguración importantes que no necesariamente son incorporados con la medición del efecto en el costo generalizado y que se vuelven cruciales en redes poco redundantes como la red carretera troncal de México y, probablemente, sea similar la situación para la mayor parte de los países de América Latina.

Por otro lado, un modelo que mida la vulnerabilidad en función de los cambios en accesibilidad está más relacionado con la medición de la inhibición de viajes que con el efecto en los tiempos de viaje, lo cual también es importante incorporar sobre todo para los traslados de mercancías, pues ello repercute de manera directa en el sistema económico completo.

Así, un modelo que mida cambios en accesibilidad podría generar las primeras aproximaciones para medir la afectación en términos de la pérdida de accesibilidad; sin embargo, podrían complementarse con la estimación de otros efectos como la pérdida de conectividad entre pares origen-destino y la afectación en el costo generalizado.

Por ello, se propone un modelo multicriterio que estime los efectos que podría producir la obstrucción de un arco en el funcionamiento de toda la red en términos de cambios en conectividad, accesibilidad y tiempo de viaje.

Queda claro que la propuesta de un modelo multicriterio, si bien atiende las diferentes perspectivas de análisis pertinentes para la toma de decisiones, introduce la complejidad adicional de valorar la relevancia de las diferentes perspectivas entre sí, pues es muy posible que los tramos críticos obtenidos por cada uno de los criterios hasta aquí revisados (topología, accesibilidad y flujos) difieran en términos de su importancia en los efectos sobre la red. Así, la propuesta ha de resolver de manera convincente la valoración de los criterios incluidos en el modelo.

\section{Aplicación propuesta en México}

Actualmente las economías nacionales dependen aún más de los sistemas de transporte por lo que sus interrupciones provocarán mayores afectaciones económicas en el futuro próximo; dicho problema será un reto para los investigadores en el área de transporte (Husdal, 2006), sobre todo para los países en desarrollo como México, cuyo sistema económico está supeditado al autotransporte, en una red nacional muy poco densa y con pocas rutas redundantes.

Por tal motivo y dada la revisión anterior, se recomienda el desarrollo de una metodología de análisis multicriterio para identificar los arcos críticos de la red, que considere las limitantes de información en México. La identificación de los arcos críticos se realizará bajo el enfoque de la cuantificación de los efectos que provoca la obstrucción de arcos individuales en el funcionamiento de la red de carreteras analizada, independientemente de la probabilidad de obstrucción de cada arco.

Es conveniente que la cuantificación de los efectos se lleve a cabo considerando los tres criterios:

a) conectividad de pares origen-destino,

b) accesibilidad,

c) tiempo de viaje. Cada arco podrá ser analizado por separado de tal forma que se puedan obtener índices de vulnerabilidad de la red para afectaciones de falla en arcos individuales. El modelo recomendado tomaría la forma siguiente:

$$
I_{a}=\mathrm{f}_{1}\left(\frac{R_{a}}{R_{0}}\right)+\mathrm{f}_{2}\left(\frac{A_{a}}{A_{0}}\right)+\mathrm{f}_{3}\left(\frac{c_{a}}{c_{0}}\right)
$$

donde:

$R_{a}=\quad$ número de rutas que cruzan el arco $a$ y conectan los pares de centroides de la red analizada,

$R_{0}=\quad$ número de rutas totales que conectan la totalidad de los pares de centroides de la red analizada,

$A_{a}=\quad$ suma de los índices de accesibilidad de todos los centroides de la red para el escenario en donde se simula la obstrucción del arco $a$,

$A_{0}=\quad$ suma de los índices de accesibilidad de todos los centroides de la red para el escenario base;

$c_{a}=\quad$ costo del tiempo de viaje de todo el sistema cuando está totalmente obstruido el arco $a$,

$c_{0}=\quad$ costo del tiempo de viaje de todo el sistema cuando están presentes todos los arcos de la red analizada, 
$\mathrm{f}_{1}=$ factor del criterio de conectividad,

$\mathrm{f}_{2}=$ factor del criterio de accesibilidad,

$\mathrm{f}_{3}=$ factor del criterio de tiempo de viaje.

El primer término del modelo recomendado, cuyo signo es positivo, plantea la vulnerabilidad de la red a un arco específico como proporcional al número relativo de rutas origen destino afectadas por su obstrucción. En este criterio, deberán ser resueltas tres indeterminaciones actuales. Una referente a la importancia de este criterio respecto a los otros dos (más adelante se aborda en conjunto con los parámetros respectivos de los otros dos criterios).

Otra definición necesaria para el término se refiere a las rutas pertinentes a considerar: sin rizos y con una relación distancia total/distancia a vuelo de pájaro "adecuada." Para ello, se recurre a la simplificación de la red total de caminos para el automotor, restringiéndola exclusivamente a la red federal troncal y algunos arcos estatales secundarios. Una tercera definición requiere tomar en cuenta las diferencias en la importancia de las rutas, ponderadas de acuerdo a la cantidad de viajes generados en sus respectivos centroides.

El signo del segundo término es negativo. La vulnerabilidad de la red a un determinado arco obstruido es inversamente proporcional a la accesibilidad relativa resultante de su obstrucción (o directamente si se trata de la impedancia).

Finalmente, se propone tomar en cuenta la capacidad de los arcos carreteros en atención al criterio de tiempo de viaje, ya que se ha argumentado (Jenelius et al., 2006; Scott et al., 2006; Tàmpere et al., 2007) que cuando una red carretera se somete a una gran canti- dad de tráfico desviado, un pequeño aumento en el flujo podría causar más daños porque estos podrían magnificarse y presentar un comportamiento en cascada a través de toda la red de carreteras hasta que probablemente ésta colapse.

Para estimar el tiempo de viaje, se tomará en cuenta el nivel de congestionamiento que ocasionaría la obstrucción de un determinado arco y se aplicará un modelo de asignación estática que tome en cuenta la capacidad de los arcos y la estimación de la demanda. ${ }^{1}$ En la tabla 1 se muestran los resultados de la aplicación de forma independiente de dos de los criterios propuestos (conectividad y tiempo de viaje) en la red ficticia de la figura 1. Adicionalmente a esta limitante, los resultados obtenidos para los dos criterios son parciales, debido a que no se han determinado valores para los factores $\mathrm{f}_{1} \mathrm{y}_{3}$ (ecuación 9); sin embargo, los valores permiten observar que los arcos 8, 14 y 17 tienen los primeros lugares en la jerarquía del criterio (a) y del (c), y que otros arcos como el 5, 6, 10, 13 y 16 tienen jerarquías muy diferentes asignadas en cada criterio, debido a que los dos criterios miden características distintas de los arcos. Estas diferencias generarían incertidumbre en la toma de decisión sobre acciones preventivas o correctivas sobre la red carretera.

\footnotetext{
${ }^{1}$ Aunque se ha argumentado que es posible obtener una mejor estimación del congestionamiento a través de la aplicación de un modelo de asignación dinámica (Tàmpere et al., 2007), no es factible su aplicación en México, debido a que no se cuenta con gran parte de la información requerida; además de que solamente se han realizado aplicaciones de dichos modelos con un enfoque de vulnerabilidad en otros países en ámbitos subnacionales (Tàmpere et al., 2007) y no en redes tan extensas como las carreteras de México.
}

Tabla 1. Resultados de la aplicación parcial de la metodología propuesta a una red ficticia

\begin{tabular}{|c|c|c|c|c|c|c|c|c|}
\hline Arco, a & $\begin{array}{l}\text { Distancia } \\
(\mathrm{km})\end{array}$ & $\begin{array}{l}\text { Velocidad } \\
(\mathrm{km} / \text { hora })\end{array}$ & $\begin{array}{c}\text { Capacidad } \\
\text { (vehículos/hora) }\end{array}$ & $\begin{array}{c}\text { Rutas que pasan } \\
\text { por el arco a } \\
\left(\mathrm{R}_{\mathrm{a}}\right)\end{array}$ & $\left(\frac{R_{a}}{R_{0}}\right)$ & $\begin{array}{c}\text { Jerarquía, } \\
\text { criterio (a) } \\
\text { conectividad }\end{array}$ & $\left(\frac{c_{a}}{c_{0}}\right)$ & $\begin{array}{l}\text { Jerarquía, } \\
\text { criterio (c) } \\
\text { tiempo de } \\
\text { viaje }\end{array}$ \\
\hline 1 & 13 & 100 & 2000 & 10 & 0.1111 & 10 & 1.0192 & 13 \\
\hline 2 & 12 & 60 & 1600 & 6 & 0.0667 & 13 & 1.0382 & 11 \\
\hline$\overline{3}$ & 17 & 80 & 1800 & 2 & 0.0222 & 15 & 1.0430 & 9 \\
\hline 4 & 13 & 100 & 2000 & 0 & 0.0000 & 17 & 1.0096 & 16 \\
\hline 5 & 13 & 80 & 1800 & 10 & 0.1111 & 9 & 1.0581 & 6 \\
\hline 6 & 17 & 60 & 1600 & 24 & 0.2667 & 1 & 1.0000 & 17 \\
\hline 7 & 12 & 80 & 1800 & 14 & 0.1556 & 7 & 1.0437 & 8 \\
\hline 8 & 17 & 120 & 2200 & 18 & 0.2000 & 2 & 1.1359 & 1 \\
\hline 9 & 16 & 120 & 2200 & 0 & 0.0000 & 16 & 1.0551 & 7 \\
\hline 10 & 12 & 100 & 2000 & 16 & 0.1778 & 5 & 1.0392 & 10 \\
\hline 11 & 12 & 60 & 1600 & 8 & 0.0889 & 11 & $1.025 \overline{2}$ & 12 \\
\hline 12 & 10 & 60 & 1600 & 4 & 0.0444 & 14 & 1.0137 & 14 \\
\hline 13 & 11 & 60 & 1600 & 6 & 0.0667 & 12 & 1.0597 & 5 \\
\hline 14 & 17 & 100 & 2000 & 16 & 0.1778 & 4 & 1.0931 & 2 \\
\hline 15 & 17 & 80 & 1800 & 16 & 0.1778 & 6 & 1.0117 & 15 \\
\hline 16 & 10 & 80 & 1800 & 12 & 0.1333 & 8 & 1.0882 & 3 \\
\hline 17 & 10 & 80 & 1800 & 18 & 0.2000 & 3 & 1.0844 & 4 \\
\hline
\end{tabular}

$R_{\mathrm{a}}$ número de rutas óptimas (en distancia) que cruzan el arco a y conectan los nodos (centroides) de la red ficticia,

$R_{0}=90$ número de rutas totales que conectan los diez nodos de la red ficticia,

$C_{a}$ costo del tiempo de viaje de toda la red cuando está totalmente obstruido el arco a,

$c_{0}$ costo del tiempo de viaje de todo la red cuando están presentes todos los arcos de la red ficticia. Fuente: Elaboración propia. 


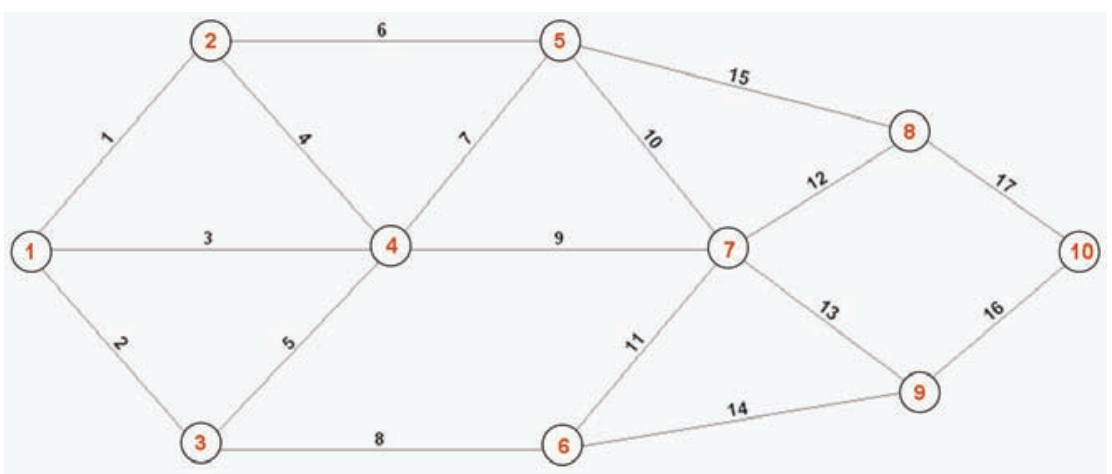

Figura 1. Red ficticia empleada para la aplicación parcial del modelo propuesto

Fuente: Elaboración propia.
Como se observa en los resultados parciales de este ejercicio (sin asignar valores relativos a cada criterio), no se obtiene una definición única de la importancia de los tramos críticos de forma contundente $\mathrm{y}$, por tanto, es necesario utilizar un método que permita valorar estos para encontrar una evaluación global de la vulnerabilidad. Para ello, se propone utilizar los métodos de decisión multicriterios que tienen como propósito (Tsoukias, 2007), ayudar al tomador de decisiones ${ }^{2}$ en todas las etapas del proceso.

Para este problema, se recomienda usar métodos para evaluar un conjunto discreto de alternativas sin incertidumbre. Para ello, se han usado tres tipos de métodos: métodos de función de valor, métodos de sobreclasificación y el método de jerarquía analítica (AHP). ${ }^{3}$

En el primer grupo (Keeney et al., 1976; Dyer, 2005), el objetivo principal es la construcción de una función de valor, la cual asigna un número en el intervalo $(0,1)$, a los resultados obtenidos por una alternativa en el conjunto de criterios. Al tener asociado a cada alternativa un valor entre 0 y 1 , se pueden ordenar éstas, de mayor a menor preferencia.

La principal desventaja de este método es la dificultad para obtener la información de preferencias. Como consecuencia, han sido desarrolladas versiones simplificadas en las cuales se sacrifica la consistencia por la simplicidad de su aplicación. Entre estos métodos destaca SMART ${ }^{4}$ (Cohon et al., 1997), con el cual se obtienen funciones lineales unidimensionales normalizadas, con un valor 1 asignado al mejor resultado y 0 al peor resultado; y una función aditiva para combinar estas últimas, en donde los pesos se asignan de manera intui-

\footnotetext{
La gran mayoría de los métodos de decisión multicriterios han sido diseñados inicialmente teniendo en mente el caso de un solo tomador de decisiones y han sido adaptados posteriormente al caso de varios tomadores de decisiones.

Otros métodos pueden encontrarse en Goicoechea et al. (1982) y Olson (1996).

Abreviación de "simple multiattribute rating technique."
}

tiva a partir de un ordenamiento de criterios con respecto a su importancia.

Los métodos de sobreclasificación (Vincke, 1992; Antún, 1994; Figueira et al., 2005), pertenecen a la escuela europea de análisis de decisiones, representada por la familia de métodos ELECTRA. Su principal característica es la sustitución de la relación de preferencia, por una relación más flexible de sobreclasificación. En esta relación, una alternativa sobreclasifica a otra (es al menos tan buena como ella), si tiene mejores resultados en la mayoría de los criterios (medido a través de una relación de "concordancia") y por otra parte, en los criterios en los cuales no se tenga un mejor resultado, éste no es muy importante (medido a través de una relación de "discordancia"). La aplicación de estos métodos involucran dos fases: en la primera se construyen las relaciones de sobreclasificación anteriormente descritas y en la segunda se "explotan" estas relaciones para obtener un subconjunto de alternativas no dominadas (alternativas que no son sobreclasificadas por otras), del cual se seleccionará posteriormente la mejor alternativa. La obtención de este subconjunto se facilita con la ayuda de la gráfica de "Kernel", ya que ésta representa visualmente las relaciones de dominación entre las diferentes alternativas. Esta gráfica se construye con un nodo por cada alternativa y con $\operatorname{arcos}(i, j)$, que se tienen, siempre y cuando la alternativa $i$ sobreclasifique a la alternativa $j$. Cuando esta gráfica no contiene ciclos dirigidos, existe siempre un "Kernel" único (Figueira et al., 2005). Ejemplos de la aplicación de esta metodología en problemas de transporte, en nuestro país, pueden verse en Arroyo y Torres (2003) y Arroyo (2006).

Otro grupo de métodos relacionados con ELECTRA, es el PROMETHEE (Brans et al., 1985), el cual se desarrolló con la idea de simplificar los primeros métodos y sobre todo tratando de disminuir el número de parámetros que tiene que obtenerse de un tomador de decisiones.

El proceso de jerarquía analítica (Saaty, 1982 y 2005), es un método que utiliza solamente comparaciones en- 
tre pares de resultados considerando cada criterio por separado y entre pares de criterios entre sí para conseguir un ordenamiento final de un conjunto de alternativas. La comparación entre pares se basa en juicios subjetivos sobre la importancia relativa de un resultado (o criterio) sobre otro. Esta importancia se traduce a una escala numérica al asignar un valor entre 1 y 9 que refleja la mayor o menor importancia considerada. Los valores usados son: uno (misma importancia); tres (moderadamente más importante); cinco (fuertemente más importante); siete (muy fuertemente más importante); y nueve (extremadamente más importante). Cuando se tiene un número grande de criterios, estos pueden ser agrupados en diferentes niveles jerárquicos, en cuyo caso las comparaciones se realizan entre pares de criterios en el mismo nivel.

Después de realizar la comparación entre cada par de criterios, se obtiene una matriz en donde cada uno de sus elementos mide la importancia relativa de un criterio con respecto al otro. La relación entre estas importancias relativas y el peso absoluto de cada criterio, conduce a un sistema de ecuaciones, cuya solución permite encontrar estos pesos y es equivalente a encontrar los valores y vectores característicos de la matriz mencionada. De la misma manera, se encuentran pesos asociados a los resultados de cada alternativa en cada uno de los criterios, los cuales se combinan con los pesos absolutos obtenidos para cada criterio para encontrar una calificación global de cada alternativa. El método ha tenido una gran aceptación, pues existen paquetes computacionales que facilitan su aplicación. Estos paquetes resuelven los sistemas de ecuaciones descritos, además de realizar pruebas de consistencia, dejando únicamente como trabajo para el tomador de decisiones las comparaciones entre pares de criterios o resultados.

En los últimos años, el proceso de jerarquía analítica ha sido el más usado cuando se trata de varios tomadores de decisiones. En la aplicación de este método se maneja siempre una fase inicial en donde se obtienen resultados por cada uno de los tomadores de decisiones involucrados. La siguiente fase toma diferentes formas: puede ser una fase de interacción buscando lograr un consenso (Bryson et al., 2000); una fase en donde se procesan las preferencias individuales, buscando obtener mediante técnicas de optimización una solución que minimice las diferencias entre ellos (Chandran et al., 2005); o una fase en donde se ponderan los resultados o las preferencias de cada tomador de decisiones para encontrar una grupal (Escobar et al., 2007).

Para el caso en cuestión, se recomienda la aplicación de este último método. Existe el inconveniente, dado el gran número de arcos, de que sería prácticamente imposible obtener comparaciones por pares y por tanto, no se recomienda utilizarlo para la fase de comparación de resultados. Sin embargo, se recomienda aplicar este método hasta el nivel de los criterios, junto con una función normalizada (posiblemente lineal) para calificar los resultados en cada uno de ellos.

\section{Conclusiones}

La metodología propuesta permitirá jerarquizar los tramos de la red federal de carreteras en México, así como la identificación de los tramos críticos. La jerarquía de los tramos carreteros podría servir como un criterio para priorizar la construcción, mantenimiento y rehabilitación de tramos carreteros en México.

\section{Agradecimientos}

Se agradece la colaboración de Eric Moreno Quintero, investigador del Instituto Mexicano del Transporte.

\section{Referencias}

Antún J.P. Toma de Decisiones Multicriterio: El Enfoque ELECTRE. Series del Instituto de Ingeniería D-38, UNAM. Marzo 1994. p. 35.

Arroyo J.A. Evaluación social de redes regionales de caminos. Metodología y estudio de caso. Publicación técnica No 298, Instituto Mexicano del Transporte, Sanfandila, Qro. 2006. P. 44.

Arroyo J.A. y Torres G. Metodología de evaluación social de proyectos de caminos rurales en México. Publicación Técnica No 234, Instituto Mexicano del Transporte, Sanfandila, Qro. 2003. P.67.

Berdica K. An Introduction to Road Vulnerability: What has Been Done, is Done and Should be Done. Transport Policy, 9(2):117127. 2002a. ISSN: 0967-070X.

Berdica K. Vulnerability: A Model-Based Case Study of the Road Network in Stockholm. TraVIS for Roads: Examples of Road Transport Vulnerability Impact Studies. Tesis (doctorado). Sweden. KTH. 2002b.

Berdica K. y Eliasson J. Regional Accessibility Analysis from a Vulnerability Perspective. Second International Symposium on Transportation Network Reliability (INSTR). Christchurch, New Zealand. Ed. Nicholson A.,Dantas A. 2004. Pp. 89-94.

Bryson N., Joseph A. Generating Consensus Priority Interval Vectors for Group Decision-making in the AHP. Journal of Multicriteria Decision Analysis, 9:127-137. 2000. ISSN: 1057-9214.

\footnotetext{
Para la aplicación del modelo multicriterio propuesto se utilizará un Sistema de Información Geográfica, así como la red de carreteras referenciada geográficamente del Inventario Nacional de Infraestructura para el Transporte, realizado por el Instituto Mexicano del Transporte.
} 
Cohon J., Rothley K. Multiobjective Methods. Design and Operation of Civil and Environmental Engineering Systems. Edited by ReVelle Ch., McGarity A. New York. John Wiley and Sons, 1997.

Chandran B., Golden B., Wasil E. Linear Programming Models for Estimating Weights in the Analytic Hierarchy Process. Computers \& Operations Research, 32(9):2235-2254. September 2005. ISSN: 0305-0548.

Di Gangi M., Luongo A.S. Measures of Network Vulnerability Indicators for Risk Evaluation and Exposure Reduction. Environmental Health Risk III. UK. Wessex Institute of Technology. 2005. ISSN: 1743-3525.

Dyer J. Maut-Multiattribute Utility Theory. Multiple Criteria Decision Analysis - State of the Art Surveys. Ed. by Figueira J., Greco S., Ehrgott M. Springer International Series in Operations Research and Management Science, Vol. 76. 2005.

Escobar M.T., Moreno-Jimenez J.M. Aggregation of Individual Preference Structures in Ahp-Group Decision Making. Group Decision and Negotiation, 16:287-301. 2007.

Figueira J., Mousseau V., Roy B. ELECTRE Methods. Multiple Criteria Decision Analysis - State of the Art Surveys. Ed. by Figueira J., Greco S., Ehrgott M. Springer International Series in Operations Research and Management Science, Vol. 78. 2005.

Goicoechea A., Hansen D.R., Duckstein L. Multiobjective Decision Analysis with Engineering and Business Applications. New York. John Wiley, 1982.

Husdal J. Transport Network Vulnerability which Terminology and Metrics Should We Use? NECTAR Cluster 1. Seminario, Molde, Norway, May 2006.

Jenelius E., Petersen T., Mattsson L. Importance and Exposure in Road Network Vulnerability Analysis. Transportation Research Part A, 40:537-560. 2006.

Keeney R.L., Raiffa H. Decisions with Multiple Objectives. New York. John Wiley, 1976.

Lleras-Echeverri G. y Sánchez-Silva M. Vulnerability Analysis of Highway Networks, Methodology and Case Study. Proceedings of the Institution of Civil Engineers, Transport, 147:223-230. 2001. ISSN: 0965-092X.

Murray-Tuite P., Mahmassani H. Methodology for Determining Vulnerable Links in a Transportation Network. Transportation Research Record: Journal of the Transportation Research Board, (1882):88-96.2004. TRB, National Research Council, Washington, DC.

Olson D. Decision Aids for Selection Problems. Standford CA. Springer-Verlag. 1996.
Saaty T.L. Decision Making for Leaders. Van Nostrand Reinhold. New York, 1982.

Saaty T.L. The Analytic Hierarchy and Analytic Network Processes for the Measurement of Intangible Criteria and for Decision-Making. In Multiple Criteria Decision Analysis - State of the Art Surveys. Ed. by Figueira J., Greco S., Ehrgott M. Springer International Series in Operations Research and Management Science, Vol. 76. 2005.

Scott D.M., Novak D., Aultman-Hall L., Guo F. Network Robustness Index: a New Method for Identifying Critical Links and Evaluating the Performance of Transportation Networks. Journal of Transport Geography, 14(3):215-227. Mayo 2006. ISSN: 0966-6923.

Tampère C., Stada J., Immers B., Peetermans E., Organe K. Methodology for Identifying Vulnerable Sections in a National Road Network. $86^{\text {th }}$ Annual Meeting of the Transportation Research Board, Washington, D.C. January 2007.

Viswanath K., Peeta S. Multicommodity Maximal Covering Network Design Problem for Planning Critical Routes for Earthquake Response. Transportation Research Record: Journal of the Transportation Research Board, (1857):1-10. 2003. TRB, National Research Council, Washington, DC.

Taylor M.A.P., D’Este G.M. Network Vulnerability: an Approach to Reliability Analysis at the Level of National Strategic Transport Networks. The Network Reliability of Transport. $1^{\text {st }}$ International Symposium on Transportation Network Reliability. Oxford, England. Ed. Bell M.G.H., Iida Y., Pergamon. 2003. pp. 23-44.

Taylor M.A.P., D'Este G.M. Critical Infrastructure and Transport Network Vulnerability: Developing a Method for Diagnosis and Assessment. Second International Symposium on Transportation Network Reliability. Christchurch, New Zealand. Ed. Nicholson A., Dantas A. 2004. pp. 96-102.

Taylor M.A.P., D’Este G.M., Sekhar S. Application of Accessibility Based Methods for Vulnerability Analysis of Strategic Road Networks. Networks and Spatial Economics, 6:267-291. Springer, 2006. ISSN: 1566-113X.

Tsoukias A. On the Concept of Decision Aiding Process: an Operational Perspective. Annals of Operations Research, 154(1):3-27. October 2007. ISSN: 1566-113X.

Vincke P. Multicriteria Decision-Aid. New York. John Wiley. 1992.- 


\section{Semblanza de los autores}

Luz Angélica Gradilla-Hernández. Obtuvo el grado de ingeniera industrial (2003) en el Instituto Tecnológico de Tepic, el grado de maestra en sistemas de transporte y distribución de carga (2005) con mención honorífica en la Universidad Autónoma de Querétaro. Se ha desempeñado como investigadora en el Instituto Mexicano del Transporte y como profesora en la Facultad de Ingeniería de la Universidad Autónoma de Querétaro, en donde actualmente estudia el doctorado en ingeniería. Su principal línea de investigación es el análisis de vulnerabilidad de redes carreteras con base en sistemas de información geográfica.

Roberto de la Llata-Gómez. Obtuvo el grado de ingeniero civil (1978) en la Universidad Autónoma de Querétaro, el grado de maestro en ingeniería con especialidad en investigación de operaciones (1980) en la Universidad Nacional Autónoma de México y el grado de doctor en ingeniería industrial (1989) en la Universidad Northwestern. Se ha desempeñado como investigador en el Instituto Mexicano del Transporte, profesor en la Universidad de las Américas-Puebla y actualmente es director del Centro Queretano de Recursos Naturales. Su principal interés de investigación es la aplicación de métodos de optimización en la solución de problemas relacionados con sistemas de transporte y de aprovechamiento de recursos hidráulicos.

Ovidio González-Gómez. Obtuvo el grado de ingeniero-arquitecto (1974) en el IPN, el grado de maestro en planeación urbana y regional (1979) en la Universidad de Illinois y el grado de doctor en desarrollo regional (1997) en la Universidad de Guadalajara. Se desempeñó durante diez años como profesor-investigador en la Universidad Autónoma Metropolitana y diez años como investigador en el Instituto Mexicano del Transporte. Desde hace diez años labora como profesor-investigador en la Universidad Autónoma de Querétaro, en donde actualmente es coordinador de la maestría en sistemas de transporte y distribución de carga. Nivel I, SNI. Su línea de investigación es el transporte y el desarrollo regional. 